\title{
Co-design de um Agente Inteligente Conversacional para Suporte ao Tele-Cuidado de Idosos em Situação de Vulnerabilidade Social e Financeira
}

\author{
Mateus de Souza Monteiro \\ Universidade Federal Fluminense \\ Niterói-RJ, Brasil \\ mateusmonteiro@id.uff.br
}

\author{
Luciana Cardoso de Castro Salgado \\ Universidade Federal Fluminense \\ Niterói-RJ, Brasil \\ luciana@ic.uff.br
}

\section{RESUMO}

Um dos principais desafios da sociedade é o envelhecimento da população. Em São Gonçalo, programas de atendimento domiciliar podem ser oferecidos para atender a idosos com mobilidade reduzida e deficiência motora. No entanto, profissionais de saúde que atendem a estes idosos sofrem com impedimento às visitas devido, entre outros fatores, ao alto índice de violência em certas áreas. Com o propósito de minimizar parte destes desafios, como por exemplo o contato entre pacientes e esses profissionais, esta pesquisa propõe o uso de Agente Inteligente Conversacional como um meio de execução do Tele-cuidado. Através de um estudo exploratório inicial foi possível identificar como os participantes abordaram as características sociais e econômicas do contexto. Além disso, os resultados também apresentaram desafios de como o Tele-cuidado é conduzido hoje e oportunidades de intervenções para mudanças nas adversidades do contexto.

\section{PALAVRAS-CHAVE}

co-design, agente inteligente conversacional, idosos, tele-cuidado

\section{INTRODUÇÃO}

Um dos principais desafios da sociedade é o envelhecimento da população [14]. O cenário desta pesquisa concentra-se em São Gonçalo, uma cidade de médio porte do Brasil, na qual os idosos sofrem com altas taxas de analfabetismo, pobreza e também deficiência motora. Com o objetivo de atender os idosos que necessitam de uma maior frequência de cuidado multiprofissional ou possuem mobilidade reduzida, atuam programas de atendimento na residência do paciente. No entanto, os profissionais de saúde que atendem estes pacientes constantemente sofrem com impedimentos às visitas, devido a fatores como a carga excessiva de trabalho, falta de materiais e o contínuo medo da violência nessa região.

Com o propósito de minimizar parte destes desafios, como o contato entre os pacientes e estes profissionais, esta pesquisa propõe o uso de Agente Inteligente Conversacional (AIC) como um meio para a execução do Tele-cuidado. Vale ressaltar que o uso de agentes

Permission to reproduce or distribute, in whole or in part, material extracted from this work, verbatim, adapted or remixed, as well as the creation or production from the content of such work, is granted without fee for non-commercial use, provided that the original work is properly credited.

IHC 2019 - WORKSHOP DE TESES E DISSERTAÇÕES, Outubro 21-25, 2019, Vitória, Brasil. In Anais Estendidos do XVIII Simpósio Brasileiro sobre Fatores Humanos em Sistemas Computacionais. Porto Alegre: SBC

(C) 2019 by the author(s), in accordance with the terms of the Creative Commons Attribution-NonCommercial 4.0 International Public License (CC BY-NC 4.0). neste cenário não propõe a substituição do contato humano, essencial para a superação da solidão e do declínio cognitivo e emocional dos idosos [5]. Em uma recente pesquisa realizada na cidade, foi apontado uma necessidade de intervenção na prevenção de complicações clínicas de idosos que sofrem com Doenças Crônicas Não Transmissíveis (DCNT), podendo o agente atuar neste cenário [4]. Entretanto, a criação de um agente inteligente deve considerar o contexto investigado [6].

Na primeira etapa desta pesquisa, conduziu-se um estudo exploratório qualitativo com a participação de profissionais de saúde que possuem experiência no cenário de atendimento a domicílio. Sabendo da necessidade do balanceamento das questões tecnológicas e sociais [1], esta primeira etapa teve por objetivo responder as seguintes questões de pesquisa: quando e quais estratégias de intervenção com agentes inteligentes poderiam fornecer prevenção de complicações clínicas a idosos com base no perfil, contexto e suas limitações de usuário?; e quais reflexões sociais, econômicas e éticas do contexto que os profissionais de saúde estão inseridos refletem no design participativo de agentes inteligentes?

Para tanto, adotou-se conceitos de co-design e práticas de Design Thinking [20] para o levantamento de informações referente às relações do cenário, uma vez que não foram encontradas metodologias específicas para co-criação de diálogos de agentes inteligentes, sem vínculo à tecnologias específicas. Além disso, esta pesquisa se diferencia de trabalhos disponíveis na literatura em diversas formas, principalmente no aspecto de envolver parte dos membros do espaço de pesquisa desde o início do desenvolvimento do agente até a fase de coleta e criação dos diálogos. Por fim, com relação aos próximos passos, cita-se a avaliação formativa do protótipo com outros especialista; a definição das metas de design; o desenvolvimento da solução com base no conhecimento levantado; e a avaliação dos fenômenos de significação e comunicação no processo de melhora da alfabetização de saúde dos cuidadores através do AIC.

\section{BASE TEÓRICA E TRABALHOS RELACIONADOS}

De acordo com Jusoh [7], um agente conversacional é uma das aplicações emergentes de processamento de linguagem natural. Os AICs podem ser aplicados em domínios como assistência médica ou até mesmo negócios online. Um grande benefício de implantar um AIC é que ele está disponível 24 horas por dia, 7 dias por semana e tem um alto potencial de reduzir o custo de contratação de pessoal humano [7]. De acordo com Denecke, Tschanz, Dorner e May [4], os agentes permitem o fornecimento de cuidados em lugares onde as pessoas não teriam acesso. As possibilidades de uso na área 
médica incluem, por exemplo, nutricionista virtual para pacientes diabéticos, no qual o chatbot faz perguntas e no final elabora uma dieta, ou um assistente eletrônico de gerenciamento de medicação ou até mesmo musicoterapia. Os AICs também podem ser aplicados na identificação de sintomas ou no diagnóstico de doenças relacionadas à idade, afirma Eschweiler e Wanner [5]. Como exemplo, os sintomas do mal de Parkinson incluem redução na percepção e distinção de odores, e distúrbios do sono, os quais podem ser identificados com a instrução de cuidadores através de um questionário. Além disso, os AICs também podem ser programados para aplicar instrumentos de avaliação geriátrica e até mesmo questões de diferentes aspectos [5].

\subsection{Desafios do uso de AIC para idosos}

Segundo Montenegro, da Costa e Righi [12], a definição de agentes conversacionais consiste em um programa de computador ou inteligência artificial capaz de segurar uma conversa com humanos através do processamento de linguagem natural. Dentre os trabalhos que fizeram revisões de literatura do uso de AIC em saúde $[2,10,19]$, no mais recente, apresentado por Montenegro, da Costa e Righi [12] em 2019, os autores buscaram os principais contextos de interação e modelos de comunicação envolvendo AIC.

As soluções de agentes na área da saúde podem ser desenvolvidas em diferentes perspectivas, seja para o paciente, seja para o profissional de saúde em atividade ou em formação [12]. Com relação às soluções voltadas para os pacientes, estas podem auxiliar no tratamento terapêutico e cognitivo, como por exemplo, no monitoramento da condição do paciente para o auxílio ao diagnóstico e também na alfabetização em saúde, como por exemplo no entendimento do tratamento, seus métodos e resultados [12]. $\mathrm{Na}$ busca pelos desafios dos AICs em saúde, 5 categorias emergem: geração de diálogo, integração com outras tecnologias, adaptação e uso de agentes conversacionais por idosos, experiência do usuário e novas abordagens de metodologia e arquitetura. Ainda segundo os autores, todos os trabalhos que buscavam solucionar os desafios de AIC para idosos [9, 13, 16, 18], focaram no contexto do paciente.

Dentre estes trabalhos, cita-se três grandes frentes: prevenção [9], treino [13] e assistência às atividades diárias [18]. No trabalho de Nikitina, Callaioli e Baez [13], os autores descrevem os requisitos, modelos e design do AIC de treino de profissionais inexperientes para reminiscência, que é a lembrança de aspectos importantes da vida dos idosos. Com relação a prevenção, AICs podem contribuir na escolha do melhor caminho para o tratamento da doença, prevenção de suicídio e até mesmo lidar com a depressão [12]. Para tanto, estes agentes devem ter acesso ao contexto e informações do paciente [12], como no trabalho de King et al. [9], no qual buscava-se a saúde do idosos através da prevenção. O design da solução foi voltado para uma população idosa com pouco rendimento e entendimento da língua principal do país. No trabalho de Shaked [16], o autor estuda o uso de avatares como aliados destes desafios, o qual permite um canal de comunicação muito mais vívido com o idoso. Já no trabalho de Tokunaga et al. [18], os autores pesquisaram a adoção dos agentes por idosos e observaram que a adoção desta tecnologia está centrada em três pontos: personalização, custo de assistentes pessoais e o fator de aprendizado para a adaptação do uso.
Os autores Montenegro, da Costa e Righi [12] afirmam que os idosos têm se mostrado como uma questão desafiadora na adoção dos AICs. Não somente isto, tratando-se desta pesquisa, existem diversas variáveis que não apenas se relacionam a critérios de domínio e infraestrutura. O contexto socioeconômico e cultural do local onde esta pesquisa foi realizada influenciará na concepção e na adoção da solução proposta para o problema. Por fim, as diferenças dos trabalhos citados com este cenário sāo: (1) o AIC está sendo conceitualizado na perspectiva do cuidador, embora com foco na qualidade de vida do idoso; (2) a solução do AIC está voltado para a prevenção de complicações clínicas de DCNT através da verificação do estado do paciente e da execução do tratamento; (3) o processo do design da solução está sendo fundamentado no contexto da pesquisa desde o início, ou seja, a escolha do caminho da intervenção (diretamente para idoso, através do cuidador ou através do familiar) é feita de maneira participativa, evitando que ocorra alguma incompatibilidade com a proposta; e por último (4), os diálogos possuem pequenos objetivos específicos.

\subsection{Design participativo dos diálogos}

Alguns dos tópicos emergidos durante a análise do trabalho de Kasckow et al. [8] foram: preocupações com confidencialidade e problemas com vocabulário e redação. No detalhamento do primeiro tópico, os autores disseram que os participantes pediram que o termo "Machucar a si mesmo " deveria ser usado em vez de "se matar". Dentre as preocupações, estão as informações fornecidas pelo paciente para o agente durante a intervenção, que consequentemente poderiam revelar suas tendências suicidas, que não são aderentes a medicamentos, e que abusam de substâncias [8]. No trabalho de Tielman, Neerincx e Brinkman [17], os autores apresentaram o projeto e a avaliação de mensagens motivacionais personalizadas para a terapia de Transtorno de Estresse Pós-Traumático (TEPT). No design participativo do agente, para cada caso, pedia-se ao especialista que escrevesse o que eles diriam ao paciente para aumentar sua motivação para continuar no tratamento. Já no trabalho de Petukhova et al. [15], utilizou-se o design participativo a partir de dados já coletados. Segundo os autores, para uma melhor compreensão dos fenômenos de interação humana e previsão de ações dos interlocutores, o primeiro passo é coletar dados dessa interação. Por fim, no trabalho dos autores Milhorat, Schlogl, Boudy e Chollet [11], os autores afirmam que as ferramentas que permitem a concepção, construção e manutenção de um sistema de diálogo são raras. As principais diferenças dos trabalhos apresentados com o este cenário são: (1) compreensão do contexto de maneira abstraída da tecnologia, pois os participantes não possuem conhecimento das ferramentas atuais; (2) não há interação humana previamente registrada e o (3) propósito é a elaboração do diálogo centrado no contexto, considerando a regionalização do vocabulário e o não uso de termos técnicos.

\section{ESTUDO}

Este estudo inicial tem como objetivo responder às duas questões de pesquisa citadas na Seção 1. Para isso foi realizado um estudo exploratório qualitativo com conceitos de co-design e práticas de Design Thinking [20]. 


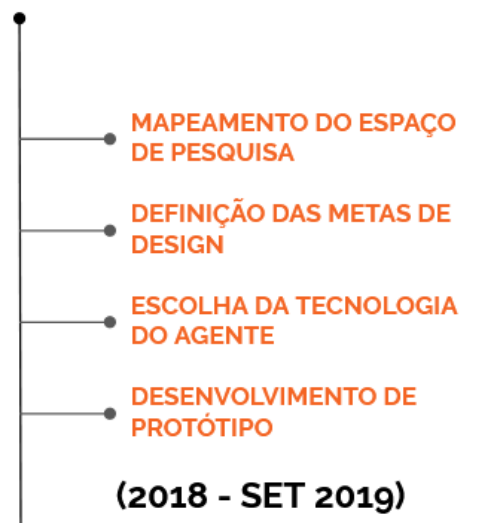

Figura 1: Passos necessários para a pesquisa. Em laranja as atividades concluídas e em magenta escuro as atividades futuras e as etapas com smiles indicam a participação de seres humanos

\subsection{Metodologia}

Dois pesquisadores em Interação Humano-Computador participaram do estudo, um no papel de mentor da discussão e outro responsável por dar suporte à dinâmica. O estudo foi dividido em três fases: Imersão, Ideação e Prototipação. Os participantes responderam o termo de consentimento livre e esclarecido desta pesquisa. Os dados empíricos coletados foram as falas e imagens dos participantes durante todo o processo do estudo. A análise dos dados seguiu o paradigma não-preditivo e interpretativo com a abordagem de codificação dos dados empíricos coletados e transcritos.

Foram adotadas duas abordagens para responder à questão de pesquisa 1: a de codificação aberta nomeando sentença por sentença e a seletiva que usa as codificações mais significativas para organizar, sintetizar, integrar e organizar grandes quantidades de dados [3]. Para análise da segunda questão, seguiu-se a abordagem de codificação top-down à luz das categorias: social, econômica e ética [1]. O estudo foi precedido por uma sessão piloto para avaliação do roteiro e da estrutura de coleta de material empírico, que não apresentou necessidade de nenhuma mudança.

Com relação aos participantes, foram selecionados dois profissionais de saúde baseado em suas experiências prévias com idosos e conhecimento do cenário. Ambos os selecionados possuem suas pesquisas voltadas para o cenário. $\mathrm{O}$ primeiro participante é estudante de enfermagem e está no nono período. Sua pesquisa de trabalho final do curso é relacionada ao monitoramento de idosos na pós-cirurgia através de um aplicativo de mensagens. Além disso, possui experiência em um projeto estadual em Tele-cuidado. O segundo participante é profissional formado em enfermagem desde 2012, especialista especialista na saúde do idoso e atualmente é mestrando. Possui experiência trabalhando em diversos cenários críticos de home care como gerente de um setor de Tele-cuidado.
Durante a fase de imersão e entendimento do contexto, foi identificado o quanto a esposa de um idoso com DCNT carece de acompanhamento. Isso se deve principalmente a uma questão cultural no perfil dos idosos masculinos atendidos, no qual, não há uma cultura de autocuidado e atenção a própria saúde. Neste caso, deixando toda a responsabilidade para a esposa, contrariando as estatísticas que indicam o perfil de filho como o mais prevalente dentre os cuidadores. Segundo os participantes, muitas vezes a esposa não tem o conhecimento técnico para realizar a função de cuidadora. Estes fatores convergiram para um dos desafios atualmente, que é a inexperiência dos cuidadores informais, o qual ocasiona Inúmeras falhas de processo e, como consequência, as complicações clínicas.

Durante a construção do diálogo, os participantes propuseram trabalho, pois, de acordo com um dos participantes, os cuidadores precisam ser reconhecidos quando executam um bom trabalho. Além disso, mensagens com teor de alerta também poderiam ser utilizadas na indicação de que algo deve ser mudado e quando há a necessidade emergencial de verificação em um posto de saúde, pois a situação já se encontra crítica. Outro ponto social identificado foi a negligência, pois existe a possibilidade do idoso ou do próprio cuidador dizer que irá fazer e acabar se esquecendo ou apenas não correspondendo com o combinado. Outra característica identificada é a necessidade de uma postura incisiva quando necessário, uma vez que a pressão não foi medida e nem a insulina ingerida, então os participantes são orientados pela mensagem "Meça agora!". Isto se deve principalmente pela necessidade de diálogos que representem clareza e não dúvida e/ou subjetividade. Com relação aos fatores éticos, os participantes reconhecem que a interação destas questões deve ser entre o profissional de saúde e o idoso, já que sentem-se felizes ao participar de uma pesquisa que tem por objetivo sua própria qualidade de vida.

\subsection{Resultados} o uso de mensagens que transmitissem valorização de um bom 


\section{CONCLUSÕES PRELIMINARES}

Os principais resultados deste estudo inicial mostraram desafios no cenário e no formato atual do Tele-cuidado. Quanto aos desafios do Tele-cuidado, cita-se a incompatibilidade de para quem a solução está sendo proposta, que pode ocasionar na falha da intervenção e inconsistência nos dados coletados; e a demanda por um entendimento profundo do cenário, pois segundo um dos participantes, em um cenário, diferentes interlocutores podem ter opiniões diferentes acerca do idoso e da situação do tratamento, demandando um esforço técnico e empático do profissional de saúde. Neste sentido, tarefas repetitivas poderiam ser suportadas por tais sistemas, disponíveis todos os dias da semana e em qualquer horário [4]. A aplicação do estudo também trouxe oportunidades de mudança e melhoria do cenário explorado. Durante a análise, foram identificadas características sociais e econômicas no momento da elaboração dos diálogos e durante a proposta das soluções. Como por exemplo, a seleção das palavras e tempos verbais adequados, utilizando frases no imperativo, quando necessário, e levantando casos em que a família não possui recursos para comprar um smartphone. Além disso, propuseram o uso de mensagens que ajudem na identificação da (in)adequação do tratamento de forma que o profissional de saúde possa orientar o caminho adequado.

\section{TRABALHOS FUTUROS}

A Figura 1 descreve as atividades que compõe os passos realizados e futuros desta pesquisa. Ao observar da esquerda para a direita, o período entre 2018 e setembro de 2019 compreendeu as fases já executadas de mapeamento do espaço de pesquisa, definição das metas de design, escolha da tecnologia do agente e o desenvolvimento do protótipo. Dentre as etapas que estão relacionadas a metodologia da pesquisa, cita-se a (1) Avaliação Formativa, no qual, com a participação de profissionais da área da saúde do contexto estudado, esta etapa compreenderá algumas atividades de avaliação do material levantado nas etapas concluídas. Esta fase abrangerá as modalidades de observação e entrevista.

Tanto a $2^{\mathrm{a}}$ como a $5^{\mathrm{a}}$ fase compreenderão a análise dos dados seguindo um paradigma não-preditivo e interpretativo com a abordagem de codificação dos dados empíricos coletados e transcritos. Serão adotadas duas abordagens: a de codificação aberta nomeando sentença por sentença e a seletiva que usa as codificações mais significativas para organizar, sintetizar, integrar e organizar grandes quantidades de dados.

Na fase (3), serão realizadas as adequações com base no resultado da fase 2 . $\mathrm{Na} 4^{\mathrm{a}}$ e última fase que contará com a participação de seres humanos, o estudo será apenas de caráter observacional, no qual os participantes testarão a aplicação em um estado mais maduro. Por fim, na $6^{\text {a }}$ fase a adequação da solução com base nos resultados da $5^{\text {a }}$ fase.

\section{AGRADECIMENTOS}

O presente trabalho foi realizado com apoio da Coordenação de Aperfeiçoamento de Pessoal de Nível Superior - Brasil (CAPES) Código de Financiamento 001.

\section{REFERÊNCIAS}

[1] M. C. C. Baranauskas, M. C. Martins, and J. A. Valente. 2013. Codesign de Redes Digitais: tecnologia e educação a serviço da inclusão social. Penso Editora, Universidade Estadual de Campinas - UNICAMP.

[2] J. Bibault, B. Chaix, P. Nectoux, and B. Brouard. 2019. Healthcare ex Machina: Are conversational agents ready for prime time in oncology? Clinical and translational radiation oncology (2019).

[3] K. Charmaz. 2014. Constructing grounded theory (2 ed.). Sage Publications, Great Britain.

[4] K. Denecke, M. Tschanz, T. Dorner, and R. May. 2019. Intelligent Conversational Agents in Healthcare: Hype or Hope? Studies in health technology and informatics 259 (2019), 77-84.

[5] Gerhard W. Eschweiler and Leo Wanner. 2018. How Can Intelligent Conversational Agents Help? The Needs of Geriatric Patients and Their Caregivers. In Intelligent Conversation Agents in Home and Geriatric Care Applications. CEUR-WS, Stockholm, Sweden, 1-9.

[6] J. J. Ferreira, A. Fucs, and V. Segura. 2019. Should I Interfere'AI-Assistants' Interaction with Knowledge Workers: A Case Study in the Oil and Gas Industry. In Extended Abstracts of the 2019 CHI Conference on Human Factors in Computing Systems. ACM, Glasgow, Scotland Uk, CS20.

[7] S. Jusoh. 2018. Intelligent Conversational Agent for Online Sales. In International Conference on Electronics, Computers and Artificial Intelligence (ECAI). IEEE, Iasi, Romania, 1-4.

[8] J. Kasckow, S. Zickmund, A. Rotondi, A. Mrkva, J. Gurklis, M. Chinman, L. Fox, M. Loganathan, B. Hanusa, and G. Haas. 2014. Development of telehealth dialogues for monitoring suicidal patients with schizophrenia: Consumer feedback. Community mental health journal 50, 3 (2014), 339-342.

[9] A. C. King, T. W. Bickmore, M.I. Campero, L.A. Pruitt, and Yin J. L. 2013. Employing Virtual Advisors in Preventive Care for Underserved Communities: Results From the COMPASS Study. Journal of Health Communication 18, 12 (2013), 1449-1464. https://doi.org/10.1080/10810730.2013.798374 PMID: 23941610.

[10] L. Laranjo, A. G. Dunn, H. L. Tong, A. B. Kocaballi, J. Chen, R. Bashir, D. Surian, B. Gallego, F. Magrabi, A. Y. S. Lau, and E. Coiera. 2018. Conversational agents in healthcare: a systematic review. Fournal of the American Medical Informatics Association 25, 9 (2018), 1248-1258.

[11] P. Milhorat, S. Schlögl, Jérôm Boudy, and G. Chollet. 2013. What if everyone could do it?: a framework for easier spoken dialog system design. In SIGCHI symposium on engineering interactive computing systems. ACM, London, United Kingdom, 217-222.

[12] J. L. Z. Montenegro, C. A. da Costa, and R. da R. Righi. 2019. Survey of conversational agents in health. Expert Systems with Applications 129 (2019), 56-67. https://doi.org/10.1016/j.eswa.2019.03.054

[13] S. Nikitina, Sara Callaioli, and M. Baez. 2018. Smart Conversational Agents for Reminiscence. CoRR abs/1804.06550 (2018). arXiv:1804.06550 http://arxiv.org/ abs $/ 1804.06550$

[14] Organização Mundial da Saúde (OMS) 2011. Saúde Global e Envelhecimento. Organização Mundial da Saúde (OMS).

[15] V. Petukhova, M. Gropp, D. Klakow, A. Schmidt, G. Eigner, S. Topf, M. Srb, P. Motlicek, B. Potard, J. Dines, O. Deroo, R. Egeler, U. Meinz, and S. Liersch. 2014. The DBOX corpus collection of spoken human-human and human-machine dialogues. Technical Report. European Language Resources Association (ELRA).

[16] N. A Shaked. 2017. Avatars and virtual agents-relationship interfaces for the elderly. Healthcare technology letters 4, 3 (2017), 83-87.

[17] M. L Tielman, M. A. Neerincx, and W. Brinkman. 2019. Design and evaluation of personalized motivational messages by a virtual agent that assists in posttraumatic stress disorder therapy. Fournal of medical Internet research 21, 3 (2019), $1-21$.

[18] S. Tokunaga, H. Horiuchi, K. Tamamizu, S. Saiki, M. Nakamura, and K. Yasuda. 2016. Deploying service integration agent for personalized smart elderly care. In 2016 IEEE/ACIS 15th International Conference on Computer and Information Science (ICIS). 1-6. https://doi.org/10.1109/ICIS.2016.7550873

[19] A. N. Vaidyam, H. Wisniewski, J. D. Halamka, M. S. Kashavan, and J. B. Torous. 2019. Chatbots and Conversational Agents in Mental Health: A Review of the Psychiatric Landscape. The Canadian fournal of Psychiatry (2019), 2-9.

[20] M. Vianna. 2012. Design thinking: inovação em negócios. Design Thinking. 